\title{
Hausse de la détection de Corynebacterium diphtheriae au Canada : de 2006 à 2019
}

\author{
KA Bernard ${ }^{1,2 *}$, AL Pacheco ${ }^{1}$, T Burdz $^{1}$, D Wiebe ${ }^{1}$
}

\section{Résumé}

Contexte : De plus en plus on utilise la spectrométrie de masse à temps de vol avec désorption/ionisation par impact laser assistée par matrice (MALDI-TOF) pour identifier les bactéries, y compris les espèces de Corynebacterium, de façon rapide, précise et peu coûteuse. Seulement trois espèces de Corynebacterium sont capables de produire la toxine diphtérique (TD) et les souches isolées peuvent être toxigènes ou non toxigènes. II semble que l'identification bactérienne plus précise fournie par les systèmes MALDI-TOF ait entraîné une hausse du nombre de demandes d'analyse des toxines soumises au Laboratoire national de microbiologie (LNM).

Objectif : Décrire le nombre d'isolats de C. diphtheriae, de C. ulcerans et de C. pseudotuberculosis identifiés et soumis au LNM entre janvier 2006 et le 30 juillet 2019, y compris leur région géographique, leur source et s'ils produisent la TD.

Méthodes : Les isolats humains ou animaux soumis au LNM pour lesquels une de ces trois espèces de Corynebacterium a été identifiée ont été analysés sur les plans de la province, la source et la toxigénicité. L'identification de l'espèce a été confirmée, puis les spécimens ont été testés par réaction en chaîne de la polymérase afin de détecter la présence de gènes tox et, si ces gènes sont détectés, de déterminer s'ils expriment la TD à l'aide de la méthode Elek modifiée. L'analyse était descriptive.

Résultats : Au cours de la période d'étude, 639 isolats de $C$. diphtheriae et 22 isolats de $C$. ulcerans ont été identifiés; aucun isolat de C. pseudotuberculosis n'a été identifié. Il y a eu une hausse d'isolats de C. diphtheriae soumis pour analyse de la TD : de huit par année en 2006 à 15 par mois en moyenne en 2019, soit une hausse de $1200 \%$ sur une période de 13,6 ans. Les demandes d'analyse provenaient principalement de l'ouest du Canada ( $n=609 / 639 ; 95 \%$ ). La plupart (638/639, 99\%) étaient des isolats humains et la plupart provenaient de sites cutanés. De ces isolats, $87 / 639(13,6 \%)$ étaient toxigènes et $552 / 639(86,4 \%)$ étaient non toxigènes. Parmi les demandes d'analyse d'isolats de $C$. ulcerans soumises, $17 / 22$ (77\%) étaient des isolats humains et cinq (23\%) étaient des isolats animaux; 10/22 (45 \%) étaient toxigènes.

Conclusion : Il y a eu une hausse marquée du nombre d'espèces de Corynebacterium soumises au LNM pour analyse de la TD. Cette hausse pourrait être attribuable à la capacité accrue d'identifier ces bactéries à l'aide des systèmes MALDI-TOF. Une surveillance continue aidera à déterminer si cette hausse découle uniquement d'une plus grande précision du diagnostic ou s'il s'agit de nouveaux pathogènes cutanés.
Cette oeuvre est mise à la disposition selon les termes de la licence internationale Creative Commons Attribution 4.0

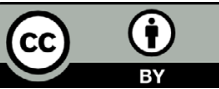

Affiliations

${ }^{1}$ Laboratoire national de microbiologie, Agence de la santé publique du Canada, Winnipeg, MB

2 Département de microbiologie médicale, Université du Manitoba, Winnipeg, MB

\section{*Correspondance:}

kathy.bernard@canada.ca

Citation proposée : Bernard KA, Pacheco AL, Burdz T, Wiebe D. Hausse de la détection de Corynebacterium diphtheriae au Canada : de 2006 à 2019. Relevé des maladies transmissibles au Canada 2019;45(11):326-31. https://doi.org/10.14745/ccdr.v45i11a04f

Mots-clés : Corynebacterium diphtheriae, espèces produisant la toxine diphtérique, rétablissement accru, MALDI-TOF

\section{Introduction}

L'infection à la Corynebacterium diphtheriae (C. diphtheriae) classique, qui cause un mal de gorge, de la fièvre et des symptômes respiratoires, est rare au Canada (1-3). Comme le vaccin contre la toxine diphtérique (TD) fait maintenant partie du schéma de vaccination systématique, moins de quatre cas de cette maladie à déclaration obligatoire sont recensés chaque année $(4,5)$. 
Dans le passé, les espèces de Corynebacterium isolées de spécimens cliniques non respiratoires n'étaient pas suffisamment analysées et déclarées. Ces bactéries étaient presque toujours considérées comme des contaminants et n'étaient pas identifiées (genre et espèce) ni soumises à des tests de sensibilité aux antimicrobiens, même si elles étaient isolées en culture pure dans des liquides organiques stériles ou des abcès de plaies profondes (3). De plus, l'identification non ambiguë du genre et de l'espèce des souches pouvait être difficile et coûteuse à réaliser à l'aide de tests phénotypiques uniquement $(1,3)$.

Il est maintenant plus largement reconnu que $C$. diphtheriae non respiratoire peut causer des infections de la peau et des plaies. La diphtérie cutanée se présente souvent sous forme d'ulcères fétides bien délimités ou de nodules à guérison lente et hautement contagieuses. Les personnes particulièrement à risque sont celles présentant des comorbidités comme le VIH, l'hépatite B ou C, le diabète, des antécédents d'ulcères récurrents, l'abus d'alcool, des antécédents de consommation de drogues injectables, les personnes qui vivent dans de mauvaises conditions socio-économiques, des refuges pour sans-abri ou des camps de réfugiés, ou qui voyagent dans des pays où ces pathogènes sont endémiques (6-11). Le traitement de la diphtérie cutanée comprend souvent l'utilisation des mêmes antibiotiques à action systémique utilisés pour le traitement des maladies respiratoires, plus la prise de précautions d'isolement (6-10) pour empêcher que les bactéries des lésions servent de réservoir et que l'infection se propage davantage $(11,12)$. Toutefois, dans certains cas, des souches de C. diphtheriae multirésistantes difficiles à traiter ont été détectées dans des cas de diphtérie cutanée (13). Ces cas entraînent parfois une septicémie et la mort. Le rôle de la vaccination préalable par I'anatoxine diphtérique dans la prévention des infections cutanées n'est pas bien compris. L'utilisation de l'antixotine n'est généralement pas recommandée, sauf en présence de signes de toxicité systémique (7).

L'identification du genre et de l'espèce de Corynebacterium a été simplifiée par l'utilisation répandue d'instruments de spectrométrie de masse à temps de vol avec désorption/ ionisation par impact laser assistée par matrice (MALDI-TOF) et de méthodes moléculaires plus précises. Les protéines sont extraites des bactéries et les empreintes digitales qui en résultent sont ensuite comparées aux entrées contenues dans la bibliothèque de données du système. Une identification très fiable des espèces peut être obtenue en quelques minutes seulement, pour moins de $1 \$$ canadien par échantillon $(14,15)$. La procédure MALDI-TOF permet d'identifier automatiquement les espèces productrices de la toxine diphtérique :

C. diphtheriae, C. ulcerans et C. pseudotuberculosis. Les infections à $C$. ulcerans et $C$. pseudotuberculosis sont rares chez les humains et sont contractées au contact d'animaux infectés. Les infections à $C$. ulcerans chez les humains sont liées au contact avec des animaux de compagnie malades, comme les chiens ou les chats, tandis que $C$. pseudotuberculosis est présent chez les moutons et les chèvres, ce qui rend les vétérinaires et les gardiens d'animaux plus à risque de contamination (3).

Au Canada, I'utilisation d'instruments MALDI-TOF a commencé en 2012 environ. Ces instruments sont maintenant largement distribués partout au pays, soit dans tous les laboratoires provinciaux de santé publique, dans de nombreux établissements hospitaliers privés/publics et dans des laboratoires vétérinaires spécialisés dans les maladies infectieuses chez les animaux. Les avantages évidents de l'utilisation systématique des systèmes MALDI-TOF sont une réduction considérable des coûts liés à la caractérisation des bactéries et l'identification beaucoup plus rapide des pathogènes comme $C$. diphtheriae. Ces avantages permettent de mettre en œuvre rapidement les traitements appropriés. Toutefois, les systèmes MALDI-TOF permettent seulement d'identifier ces bactéries et non d'établir si l'une de ces espèces de Corynebacterium produit la TD. Depuis quelques années, le Laboratoire national de microbiologie (LNM) reçoit un plus grand nombre de demandes d'analyse de la TD de laboratoires de santé publique et de laboratoires vétérinaires partout au pays. L'objectif de cette étude était de décrire le nombre d'échantillons de $C$. diphtheriae, de $C$. ulcerans et de C. pseudotuberculosis soumis au LNM entre janvier 2006 et le 30 juillet 2019, leurs caractéristiques, ainsi que le pourcentage ayant produit la TD.

\section{Méthodes}

\section{Examen des dossiers}

Les dossiers de laboratoire datés de 2006 au 30 juillet 2019 de l'unité de bactériologie spéciale (UBS) du LNM ont été examinés afin de relever les demandes d'analyse de la TD. Les renseignements examinés comprenaient la province, le site d'infection (si connu), l'espèce de Corynebacterium produisant la TD et la toxigénicité de la souche. Les demandes d'analyse reçues entre janvier et juillet 2019 ont été examinées de manière plus approfondie afin de connaître la source de la souche, car ces données faisaient normalement partie des demandes d'analyse les autres années. Les renseignements sur les antécédents de vaccination et les antécédents médicaux du patient étaient généralement rares ou absents.

\section{Aperçu des types de spécimens reçus}

Depuis 2011, le Laboratoire provincial Cadham (laboratoire de santé publique du Manitoba) ne soumet au LNM que les souches pour lesquelles un test par réaction en chaîne de la polymérase (RCP) a permis de détecter des gènes tox (D. Alexander, communication personnelle, 24 juillet 2019). D'autres centres provinciaux transmettent au LNM toutes les souches qui pourraient exiger une analyse de la TD, car leurs propres laboratoires n'effectuent pas ce type d'analyse. Le LNM reçoit des cultures pures d'isolats de bactéries isolées par des laboratoires provinciaux de santé publique ou par leurs clients, puis transmis au LNM par les laboratoires pour analyse des 
toxines. Des souches sont reçues de laboratoires vétérinaires à l'occasion.

\section{Confirmation des isolats de Corynebacterium diphtheriae}

De 2006 à 2018 environ, le phénotype des souches de I'UBS était analysé à partir d'un panel API CORYNE (bioMérieux, France), afin d'identifier l'espèce et d'aider à classer l'isolat dans un des quatre biovars de $C$. diphtheriae (c'est-à-dire les biotypes gravis, mitis, belfanti et intermedius) $(1,3)$. Le panel API CORYNE a identifié formellement $C$. diphtheriae dans les souches précédemment caractérisées à l'aide de la méthode de séquençage du gène $A R N r 16 S$ avec test de référence; le LNM utilise aussi des méthodes moléculaires pour corroborer l'identification des espèces au besoin. Le LNM a cessé d'effectuer la caractérisation des souches de biovars en mai 2019, sauf sur demande. L'isolat de Corynebacterium diphtheriae identifié a été validé par le LNM pour utilisation dans la bibliothèque Bruker Microflex et Biotyper, car cette méthode permet aussi d'identifier formellement les espèces (données non montrées).

\section{Identification d'isolats de Corynebacterium ulcerans et de C. pseudotuberculosis}

Ces espèces se distinguent facilement des autres par le genre Corynebacterium, y compris $C$. diphtheriae, mais sont difficiles à différencier des autres à l'aide des méthodes de laboratoire courantes (1). Les phénotypes de ces espèces, établis à partir de sucres traditionnels ou de panels API CORYNE, sont très similaires $(1,2)$. Ces espèces sont similaires à $99,4 \%$ selon la méthode de séquençage des gènes ARNr 16S, trop similaires pour pouvoir les différencier clairement les unes des autres, et peuvent produire des résultats similaires à l'aide des systèmes MALDI-TOF (données non montrées) (1). Par conséquent, I'identification précise de ces espèces est corroborée par le LNM grâce à un séquençage partiel du gène rpoB (16).

\section{Détection des gènes tox cibles de la diphtérie et expression de la toxine diphtérique}

L'UBS effectuait des tests RCP classiques pour détecter des gènes tox cibles (13), mais elle s'est tournée vers des tests RCP en temps réel en 2018. Le test RCP en temps réel permet de détecter le gène tox et une partie du gène rpoB spécifique à C. diphtheriae à l'aide de la plateforme Quant Studio (Applied Biosystems) $(17,18)$. La souche toxigène, $C$. diphtheriae ATCC 19409 (NCTC 3984), et la souche non toxigène, C. xerosis ATCC 373T, ont été utilisées comme contrôles positif et négatif, respectivement. L'expression de la TD a été détectée à l'aide du test Elek modifié uniquement pour les souches pour lesquelles le test RCP a permis de détecter des gènes tox $(19,20)$. Les résultats positifs du test Elek peuvent être déclarés dans les $48 \mathrm{~h}$ ou dès que l'expression est visuellement détectée, et les résultats négatifs peuvent être déclarés après 48 heures. Les isolats pour lesquels le gène tox est détecté grâce au test PCR mais qui n'expriment pas la TD à la suite du test Elek sont déclarés non toxigènes (20); ces cas sont rares au Canada, représentant $8 \%$ ou moins de toutes les souches contenant le gène tox (21).

\section{Résultats}

Au cours de la période d'étude, un nombre total de 639 isolats de C. diphtheriae ont été identifiés, dont 638/639 (99\%) provenant d'humains et $1 / 639(1 \%)$ d'un cheval. Vingt-deux isolats $(n=17 ; 77 \%$ provenant d'humains et $n=5 ; 23 \%$ provenant d'animaux) de $C$. ulcerans ont été identifiés, et aucun isolat de C. pseudotuberculosis n'a été identifié.

\section{Corynebacterium diphtheriae}

Entre 2006 et 2012, un nombre moyen de quatorze isolats de $C$. diphtheriae ont été soumis pour analyse chaque année. Entre 2013 et 2015 (soit la période coïncidant avec la hausse de l'utilisation de la technique MALDI-TOF), le nombre de demandes d'analyse annuelle était de 40 en moyenne. Entre 2016 et 2018, le nombre de demandes d'analyse a augmenté à 115 . Entre janvier et juillet 2019, un nombre moyen de 15 à 16 demandes d'analyse ont été reçues au LNM chaque mois : en extrapolant, cela représente environ 185 demandes pour cette année. Si l'on compare la période avant l'utilisation de la technique MALDI-TOF pour l'analyse des toxines de huit souches (en 2006) aux 185 demandes d'analyse prévues pour 2019, cela représente une hausse de $1200 \%$ ou 12 fois plus au cours de la période d'étude (figure 1).

Figure 1 : Demandes d'analyse de toxines pour Corynebacterium diphtheriae par année, par nombre de souches toxigènes

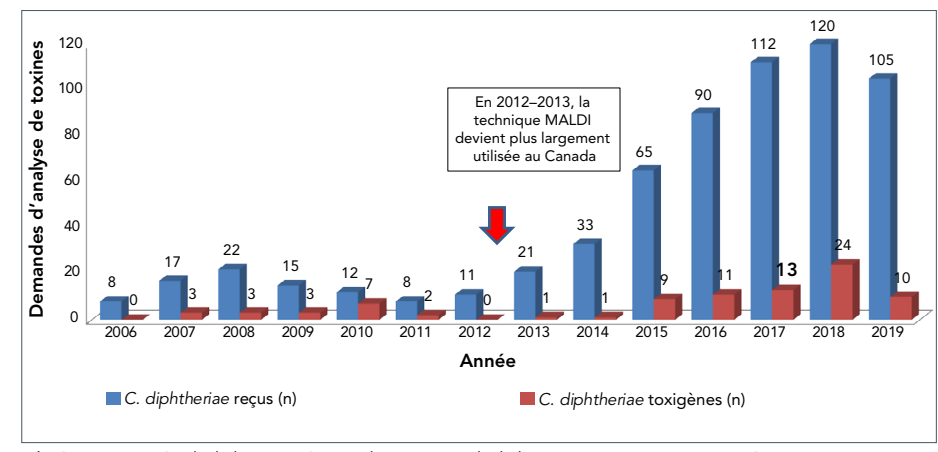

Abréviations : C. diphtheriae, Corynebacterium diphtheriae; MALDI, spectrométrie avec désorption/ionisation par impact laser assistée par matrice

Les souches de $C$. diphtheriae identifiées provenaient principalement de l'ouest du Canada ( $n=609 / 639 ; 95 \%)$, plus précisément de la Colombie-Britannique, de l'Alberta, de la Saskatchewan et du Manitoba, et un plus petit nombre de 
I'Ontario, du Québec et du Nouveau-Brunswick et de l'extérieur du Canada (figure 2).

Figure 2 : Demandes d'analyse de Corynebacterium diphtheriae et de $C$. ulcerans par province

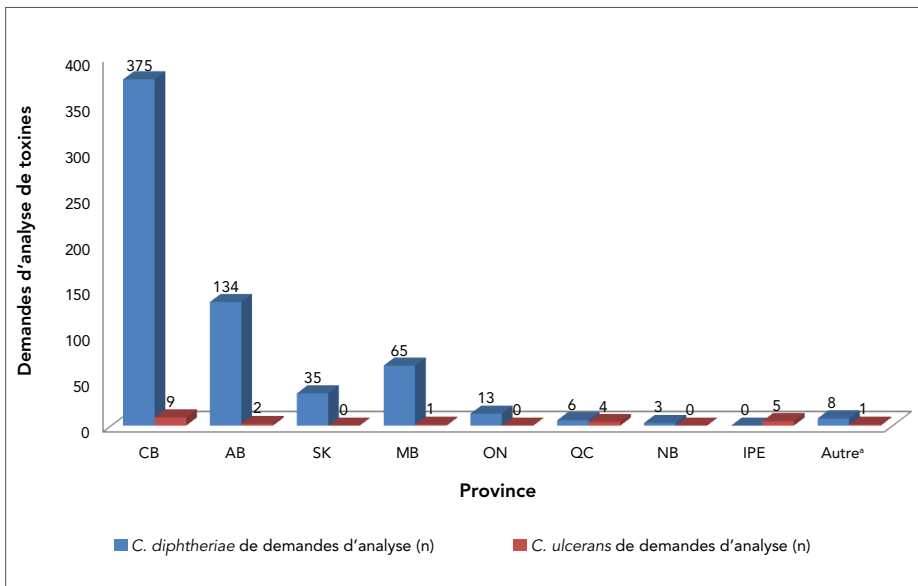

Abréviations : $\mathrm{AB}$, Alberta; $\mathrm{CB}$, Colombie-Britannique; C. Corynebacterium; IPE, Île-du-PrinceÉdouard; MB, Manitoba; NB, Nouveau-Brunswick; ON, Ontario; OC, Québec; SK, Saskatchewan Autre, sources à l'extérieur du Canada

Les demandes d'analyse de $C$. diphtheriae par province étaient les suivantes (\% de $\mathrm{n}=631$ ) : Colombie-Britannique, 375 (59\%); Alberta, 134 (21\%); Saskatchewan, 35 (5\%); Manitoba, 65 (10\%); Ontario, 13 (2\%); Québec, six (0,9\%); et Nouveau-Brunswick, trois $(0,4 \%)$. Aucune demande d'analyse de C. diphtheriae n'a été reçue de la Nouvelle-Écosse, de l'Île-du-Prince-Édouard et de Terre-Neuve-et-Labrador, et huit (1\%) demandes ont été reçues de l'extérieur du Canada. Les demandes d'analyse de $C$. ulcerans par province étaient les suivantes (\% de $n=22)$ : Colombie-Britannique, neuf $(41 \%)$; Alberta, deux (9\%); Manitoba, une (5\%); Québec, quatre (18\%); et île-du-Prince-Édouard, cinq ( $23 \%$, toutes d'isolats d'animaux). Aucune demande d'analyse de souche n'a été reçue de la Saskatchewan, de l'Ontario, du Nouveau-Brunswick, de la Nouvelle-Écosse ou de Terre-Neuve-et-Labrador.

La plupart des 105 souches de $C$. diphtheriae soumises en 2019 ont été obtenues à partir d'infections cutanées. Quatre-vingt-neuf (85\%) spécimens provenaient de sites cutanés, notamment de l'abdomen et d'abcès/plaies à la cheville, au bras, au coude, au pied, au doigt, à la tête ou au crâne, à la hanche, à la jambe, au genou, au menton, à la cuisse, au pouce, au tibia et à l'orteil; 16 spécimens (15\%) provenaient de sites non cutanés, notamment de culture sanguine $(n=1)$, de l'utérus $(n=1)$, de l'oreille $(n=2)$, des sinus, du lavage des sinus, de plaies nasales et du nez $(n=5)$, de la gorge $(n=4)$, d'expectorations $(n=1)$ et d'origine inconnue $(n=2)$.

En 2019, 10 souches toxigènes ont été isolées à partir du nez ou de sites cutanés. Ces demandes d'analyse provenaient de l'Alberta $(n=8)$, de la Colombie-Britannique $(n=1)$ et du Manitoba $(n=1)$. Aucun patient ne présentait les symptômes classiques de la diphtérie respiratoire.

\section{Corynebacterium ulcerans}

Un petit nombre de demandes d'analyse $(n=22$, figure 3$)$ ont permis d'identifier C. ulcerans. Dix-sept (77\%) spécimens étaient d'origine humaine, obtenus à partir de pus ou de la peau (plaie au bras, au coude, au pied, à la jambe ou à l'orteil). Cinq spécimens étaient d'origine animale, c'est-à-dire d'un vison $(n=1$, poumon), d'un chien ( $n=1$, oreille), d'un chat $(n=1)$ et d'un cheval ( $n=2$, abcès, peau). Ce type d'analyse a aussi augmenté d'environ $35 \%$ entre 2006 et 2012 et entre 2013 et 2019 (figure 3). Fait très important, même si leur nombre était faible, nous avons constaté que 10/22 (45\%) des souches de C. ulcerans produisaient la TD.

Figure 3 : Demandes d'analyse de toxines pour Corynebacterium ulcerans par année, par nombre de souches toxigènes

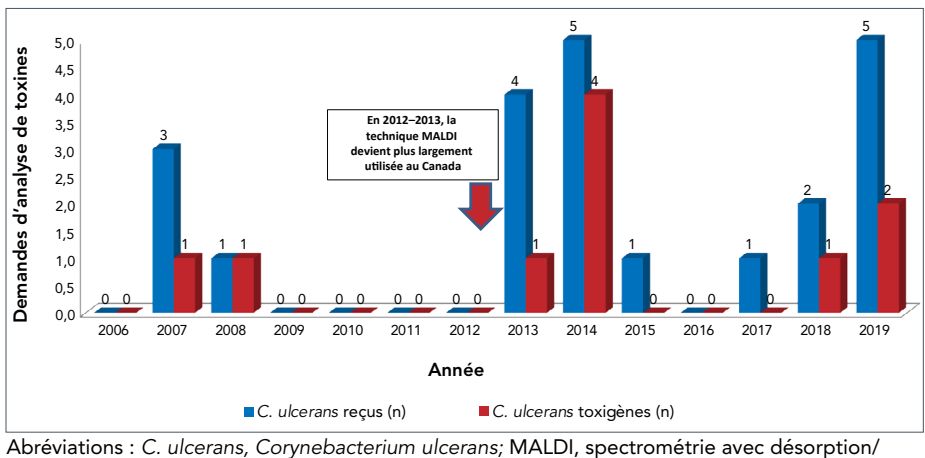

Abréviations : C. ulcerans, Corynebacterium ulcerans; MALDI, spectrométrie avec désorption/ ionisation par impact laser assistee par matrice

Notes de bas de page : Cinq des souches de C. ulcerans pour 2019 ont été reçues d'un laboratoire vétérinaire. Les 17 autres spécimens ont été obtenus de sources humaines

\section{Discussion}

Le LNM a documenté une hausse de $1200 \%$ ou 12 fois plus de demandes d'analyse de la TD pour des spécimens de Corynebacterium en moins de 15 ans. La plupart sont des souches de $C$. diphtheriae non toxigènes d'origine cutanée chez des humains, isolées en culture pure ou co-isolées en même temps que d'autres bactéries Gram positif (6). Même si leur nombre était faible dans l'ensemble, une augmentation de $35 \%$ des demandes d'analyse pour $C$. ulcerans a été enregistrée, incluant des souches provenant d'humains et d'animaux. Des hausses similaires pour $C$. diphtheriae et $C$. ulcerans ont été déclarées dans d'autres pays, notamment aux États-Unis (7-11). Cette hausse est probablement attribuable en partie à I'utilisation de plus en plus répandue des systèmes MALDI-TOF et à la précision des méthodes d'identification moléculaire. Elle pourrait également indiquer que $C$. diphtheriae est un pathogène émergent pour les infections non respiratoires.

\section{Facteurs cliniques}

Les cliniciens canadiens doivent savoir qu'il est de plus en plus courant d'isoler $C$. diphtheriae et $C$. ulcerans chez certains patients et dans certains types de spécimens. Les souches doivent être analysées pour savoir si elles sont toxigènes et, si oui, une intervention des services de santé publique, incluant 
I'analyse et la surveillance des contacts des patients, pourrait être requise en plus des traitements habituels (5).

Un antibiogramme de ces espèces est rarement requis mais pourrait s'avérer utile. Dans un article récent, nous avons décrit les résultats d'un test de susceptibilité antimicrobienne d'après une étude auxiliaire de 195 souches de $C$. diphtheriae et de 20 souches de $C$. ulcerans (22). Bien que ces espèces soient habituellement sensibles aux antibiotiques de première intention (22), la souche occasionnelle de $C$. diphtheriae s'est révélée résistante à la pénicilline ou résistante à plusieurs médicaments $(13,23)$; l'azithromycine pourrait être indiquée dans certains cas (10). Il convient de remarquer que la définition de maladie à déclaration obligatoire pour la diphtérie au Canada comprend C. diphtheriae, ainsi que C. ulcerans et C. pseudotuberculosis et, si la souche est isolée chez un patient symptomatique, elle sera considérée comme un cas confirmé (5). La diphtérie cutanée sera aussi considérée comme un cas confirmé si une souche toxigène est isolée à partir d'une plaie ou d'un site cutané (5).

\section{Limites}

Les résultats contenus dans le présent document comportent des limites inhérentes. Les laboratoires ne sont pas tenus de transmettre ces taxa au LNM aux fins de caractérisation. Par exemple, le Laboratoire provincial Cadham a lui-même analysé la TD par test PCR pour plus de 600 souches de $C$. diphtheriae durant les six premiers mois de 2019 , et aucune ne présentait de gènes tox ( $D$. Alexander, communication personnelle, 24 juillet 2019). Comme ces analyses n'ont pas été effectuées par le LNM, les données du LNM contenues dans le présent document sont sous-estimées.

Pour pouvoir isoler C. diphtheriae ou C. ulcerans dans divers spécimens cliniques, en particulier à partir de sites cutanés, les laboratoires locaux doivent être en mesure de sélectionner et de reconnaître ces agents dans la flore présente dans les biomes des sites cutanés ou respiratoires infectés. Un algorithme pourrait aider à déterminer les souches putatives de $C$. diphtheriae ou de C. ulcerans (1). En outre, il est difficile d'établir si l'absence de souches révélée par le test de la TD dans le centre et l'ouest du Canada signifie une absence de cas, ou si ces cas sont plus rares dans l'ouest du pays. Cela pourrait refléter l'utilisation de différents documents d'orientation ou de différentes pratiques de laboratoire.

\section{Recherche future}

Idéalement, les prochaines étapes pourraient comprendre des recherches cliniques et en laboratoire, notamment l'évaluation de la diphtérie cutanée à titre d'infection émergente et la collecte de données cliniques plus complètes sur les traitements et leurs résultats. D'autres recherches en laboratoire sur ces agents comprennent l'élaboration consensuelle d'un système de typage national et international pour la caractérisation de ces souches au niveau de la séquence génomique entière, qui serait utilisé pour faire le suivi in silico des souches et pour mieux caractériser leur virulence.

\section{Conclusion}

En moins de 15 ans, on a assisté à une hausse marquée du nombre de demandes d'analyse de la TD pour $C$. diphtheriae et C. ulcerans au Canada. Cela pourrait être attribuable d'une capacité accrue d'identifier ces agents à l'aide des systèmes MALDI-TOF. Une surveillance continue de ces souches de bactéries aidera à déterminer si la hausse de demandes d'analyse au LNM découle uniquement d'une plus grande précision du diagnostic ou s'il s'agit de nouveaux pathogènes cutanés.

\section{Déclaration des auteurs}

Le manuscrit a été rédigé et les données ont été révisées par K. A. B. Tous les essais techniques et la révision du manuscrit ont été effectués par A. L. P., T. B. et D. W.

\section{Conflit d'intérêts}

Aucun.

\section{Remerciements}

Nous aimerions remercier les centres suivants qui ont envoyé des souches pour étude au Laboratoire national de microbiologie : Dr L. Hoang, Centre de contrôle des maladies de la C.-B., Vancouver (Colombie-Britannique); Dr G. Tyrell et S. Gee, laboratoire provincial, Edmonton (Alberta); R. Kitzel, R. Romanow, laboratoire provincial, Regina (Saskatchewan); Dr P. Van Caeseele et Dr D. Alexander, Laboratoire Cadham, Winnipeg (Manitoba); Dr J. Kus, Santé publique Ontario, Toronto Ontario (Ontario); Dr H. Dick, Laboratoires Ideex, Richmond Hill (Ontario); Dr M. C. Domingo, Laboratoire de santé publique du Québec, Sainte-Anne-de-Bellevue (Québec); le personnel de l'Hôpital de Moncton, Moncton (Nouveau-Brunswick); le personnel de l'Hôpital Georges-Dumont, Moncton (NouveauBrunswick); M. Saab, Atlantic Veterinary Hospital, Charlottetown (Île-du-Prince-Édouard). Merci à nos étudiants, anciens et actuels, A. Vachon, D. Kielich et J. Ung, pour leurs services techniques.

\section{Financement}

Les activités de base de cette étude ont été financées par l'Agence de la santé publique du Canada. 


\section{Références}

1. Bernard KA. Coryneform Gram-Positive Rods. In: Carroll KC, Pfaller MA, Landry ML, McAdam AJ, Patel R, Richter SS, et al., editors. Manual of Clinical Microbiology. 12th ed. Washington, DC: ASM Press; 2019. p. 488-534.

2. Bernard KA, Funke G. Genus I. Corynebacterium Lehmann and Neumann 1896, 350AL emend. Bernard, Wiebe, Burdz, Reimer, Ng, Singh, Schindle and Pacheco 2010, 877. In: Whitman WB, Goodfellow M, Kämpfer P, Busse H-, Trujillo $\mathrm{ME}$, Ludwig W, et al, editors. Bergeys Manual of Systematic Bacteriology. Volume 5: The Actinobacteria. 2nd ed. New York, NY: Springer; 2012. p. 245-89.

3. Bernard K. The genus corynebacterium and other medically relevant coryneform-like bacteria. J Clin Microbiol 2012 Oct;50(10):3152-8. DOl PubMed

4. Agence de la santé publique du Canada. Maladies pouvant être prévenues par la vaccination-Dipthérie. ASPC; 2018. https://www.canada.ca/fr/sante-publique/services/ immunisation/maladies-pouvant-etre-prevenues-vaccination/ diphterie/professionels.html

5. Agence de la santé publique du Canada Dipthérie. Surveillance. ASPC; 2018. https://www.canada. $\mathrm{ca} / \mathrm{fr} /$ sante-publique/services/immunisation/ maladies-pouvant-etre-prevenues-vaccination/diphterie/ professionels/definition-nationale-cas.html

6. Lowe CF, Bernard KA, Romney MG. Cutaneous diphtheria in the urban poor population of Vancouver, British Columbia, Canada: a 10-year review. J Clin Microbiol 2011 Jul;49(7):2664-6. DOI PubMed

7. Griffith J, Bozio CH, Poel AJ, Fitzpatrick K, DeBolt CA, Cassiday P, Kenyon C, Smelser C, Vagnone PS, Culbreath K, Acosta AM. Imported Toxin-Producing Cutaneous Diphtheria - Minnesota, Washington, and New Mexico, 2015-2018. MMWR Morb Mortal Wkly Rep 2019 Mar;68(12):281-4. DOI PubMed

8. Meinel DM, Kuehl R, Zbinden R, Boskova V, Garzoni C, Fadini $D$, Dolina M, Blümel B, Weibel T, Tschudin-Sutter $S$, Widmer AF, Bielicki JA, Dierig A, Heininger U, Konrad R, Berger A, Hinic V, Goldenberger D, Blaich A, Stadler T, Battegay $M$, Sing A, Egli A. Outbreak investigation for toxigenic Corynebacterium diphtheriae wound infections in refugees from Northeast Africa and Syria in Switzerland and Germany by whole genome sequencing. Clin Microbiol Infect 2016 Dec;22(12):1003.e1-8. DOI PubMed

9. Reynolds GE, Saunders H, Matson A, O'Kane F, Roberts SA, Singh SK, Voss LM, Kiedrzynski T. Public health action following an outbreak of toxigenic cutaneous diphtheria in an Auckland refugee resettlement centre. Commun Dis Intell Q Rep 2016 Dec;40(4):E475-81. PubMed

10. Morgado-Carrasco D, Riquelme-Mc Loughlin C, Fustá-Novell X, Fernández-Pittol MJ, Bosch J, Mascaró JM Jr. Cutaneous Diphtheria Mimicking Pyoderma Gangrenosum. JAMA Dermatol 2018 Feb;154(2):227-8. PubMed
11. Coen M, Alberto C, Éperon G, Cherkaoui A, Schrenzel J. [Diphtheria: new clinical presentations of an old disease]. Rev Med Suisse 2019 Apr;15(646):786-90. PubMed

12. de Benoist AC, White JM, Efstratiou A, Kelly C, Mann G, Nazareth B, Irish CJ, Kumar D, Crowcroft NS. Imported cutaneous diphtheria, United Kingdom. Emerg Infect Dis 2004 Mar;10(3):511-3. DOl PubMed

13. Mina NV, Burdz T, Wiebe D, Rai JS, Rahim T, Shing F, Hoang $L$, Bernard K. Canada's first case of a multidrug-resistant Corynebacterium diphtheriae strain, isolated from a skin abscess. J Clin Microbiol 2011 Nov;49(11):4003-5. DOl PubMed

14. Barberis $C$, Almuzara M, Join-Lambert $O$, Ramírez MS, Famiglietti A, Vay C. Comparison of the Bruker MALDI-TOF mass spectrometry system and conventional phenotypic methods for identification of Gram-positive rods. PLoS One 2014 Sep;9(9):e106303. DOI PubMed

15. Patel R. Matrix-assisted laser desorption ionization-time of flight mass spectrometry in clinical microbiology. Clin Infect Dis 2013 Aug;57(4):564-72. DOI PubMed

16. Khamis A, Raoult D, La Scola B. rpoB gene sequencing for identification of Corynebacterium species. J Clin Microbiol 2004 Sep;42(9):3925-31. DOI PubMed

17. Schuhegger R, Lindermayer $M$, Kugler R, Heesemann J, Busch U, Sing A. Detection of toxigenic Corynebacterium diphtheriae and Corynebacterium ulcerans strains by a novel real-time PCR. J Clin Microbiol 2008 Aug;46(8):2822-3. DOI PubMed

18. De Zoysa A, Efstratiou A, Mann G, Harrison TG, Fry NK. Development, validation and implementation of a quadruplex real-time PCR assay for identification of potentially toxigenic corynebacteria. J Med Microbiol 2016 Dec;65(12):1521-7. DOl PubMed

19. Engler KH, Glushkevich T, Mazurova IK, George RC, Efstratiou A. A modified Elek test for detection of toxigenic corynebacteria in the diagnostic laboratory. J Clin Microbiol 1997 Feb;35(2):495-8. PubMed

20. Brown C; Diphtheria Guidelines Working Group. Public health control and management of diphtheria (in England and Wales). 2015 Guidelines. London, UK: Public Health England; 2015. https://assets.publishing.service.gov.uk/ government/uploads/system/uploads/attachment_data/ file/774753/Diphtheria_Guidelines_Final.pdf

21. DeWinter LM, Bernard KA, Romney MG. Human clinical isolates of Corynebacterium diphtheriae and Corynebacterium ulcerans collected in Canada from 1999 to 2003 but not fitting reporting criteria for cases of diphtheria. J Clin Microbiol 2005 Jul;43(7):3447-9. DOI PubMed

22. Bernard KA, Pacheco AL. In vitro Activity of 22 Antimicrobial Agents against Corynebacterium and Microbacterium species referred to the Canadian National Microbiology Laboratory. Clin Microbiol Newsl 2015;37(23):187-98. DOI

23. FitzGerald RP, Rosser AJ, Perera DN. Non-toxigenic penicillin-resistant cutaneous $C$. diphtheriae infection: a case report and review of the literature. J Infect Public Health 2015 Jan-Feb;8(1):98-100. DOI PubMed 